\title{
Long non-coding RNA H19 regulates LASP1 expression in osteosarcoma by competitively binding to miR-29a-3p
}

\author{
HAO JIN $^{1}$, HUAN WANG ${ }^{1}, \mathrm{XIN} \mathrm{JIN}^{2}$ and WENBO WANG ${ }^{1}$ \\ Departments of ${ }^{1}$ Orthopaedics and ${ }^{2}$ Ophthalmology, The First Affiliated Hospital of Harbin Medical University, \\ Harbin, Heilongjiang 150001, P.R. China
}

Received March 1, 2021; Accepted June 17, 2021

DOI: $10.3892 / o r .2021 .8158$

\begin{abstract}
A prevalent type of bone tumor, osteosarcoma (OS) is prone to pulmonary metastasis, which results in a high relapse risk and poor prognosis for patients. The progression of OS is significantly associated with the expression of long non-coding (lnc)RNA H19. To the best of our knowledge, however, the exact molecular mechanism of this IncRNA has not been fully investigated. The present study verified the effect of H19 on the proliferation and invasion of osteosarcoma cells via in vivo and in vitro experiments, including Cell Counting Kit-8, western blot, reverse transcription-quantitative PCR, wound healing and Transwell assays. H19 was found to be overexpressed in OS compared with corresponding normal adjacent tissue. In addition, H19 served as a competing endogenous ncRNA targeting microRNA-29a-3p and activating LIM and SH3 domain protein 1 and modulating the OS cell phenotype. The results of the present study may improve understanding of OS pathogenesis.
\end{abstract}

\section{Introduction}

Osteosarcoma (OS) is the most common malignant bone tumor that occurs in the metaphysis of long bones in adolescents; the global annual incidence of OS is 1-3 cases per million individuals (1). The clinical characteristics are atypical symptoms at onset, high degree of malignancy, distant metastasis at the early stage and poor prognosis. The current treatment options for OS include neoadjuvant chemotherapy, radiation

Correspondence to: Professor Wenbo Wang, Department of Orthopaedics, The First Affiliated Hospital of Harbin Medical University, 23 Youzheng Road, Harbin, Heilongjiang 150001, P.R. China

E-mail: hydggzh@163.com

Professor Xin Jin, Department of Ophthalmology, The First Affiliated Hospital of Harbin Medical University, 23 Youzheng Road, Harbin, Heilongjiang 150001, P.R. China

E-mail: 615122056@qq.com

Key words: long non-coding RNA H19, microRNA-29a-3p, LIM and $\mathrm{SH} 3$ domain protein 1 , osteosarcoma and surgery, however the clinical prognostic rate has not been significantly improved by these therapies. It was previously reported that the primary explanation for the poor clinical prognosis of this disease is that the current treatment methods do not effectively inhibit distant metastasis; furthermore, drug resistance and recurrence are commonly observed in patients with OS (2). In addition, the pathogenesis of OS remains unclear. The rapid development of advanced molecular biology methods as immunotherapy and targeted therapy has enabled the application of new technologies to investigate the pathogenesis of OS and provide novel therapeutic strategies for this disease (3).

Recently, numerous molecular markers have been identified for the characterization and prognostic determination of OS (4). Among these markers, several long non-coding (lnc)RNAs, which regulate numerous cellular processes, including those involved in OS progression, may represent novel therapeutic targets (5-8). Several studies have shown that lncRNAs serve key roles in multiple cellular processes, such as proliferation and apoptosis, by regulating the expression of microRNAs (miRNAs/miRs) (9-12). The lncRNA H19 has been identified as a promising miR regulator that may also be involved in the progression of OS (13). The fundamental mechanisms of H19 in OS have not been fully investigated but may be associated with the regulation of cell proliferation and invasion.

Animals, plants and several viruses contain miRs, which serve specific post-transcriptional regulatory roles in gene expression (14-16). miRs affect the proliferation, invasion and metastasis of OS, as well as tumor angiogenesis $(17,18)$. miR-29a has been reported as a tumor suppressor in breast cancer, oral squamous cell and hepatocellular carcinoma and acute myeloid leukemia (19-22). Although the roles of miRs in regulating target genes and some of their regulatory mechanisms have been determined, their contributions to the development of specific diseases remain unclear (23). The investigation of their function and pathogenic mechanisms may provide novel strategies for the treatment of OS.

LIM and SH3 domain protein 1 (LASP1) is a specific focal adhesion protein originally identified in axillary metastatic lymph nodes of patients with breast cancer. Recent studies have shown that LASP1 is involved in many biological and pathological processes (24-26). It is highly expressed in a variety of cancers, such as breast, gallbladder, colorectal, 
non-small cell lung and ovarian cancer (27-30). It is associated with poor clinical prognosis and is a new oncogene (31). Li et al (30) demonstratroved that high expression of LASP1 is associated with metastasis and poor prognosis of gallbladder cancer. In vitro experiments found that the lack of LASP1 inhibits cell cycle progression and cell migration and the overexpression of LASP1 is also associated with poor overall survival rate of patients with liver cancer (32). In human liver cancer specimens, the level of LASP1 mRNA can be used as a potential indicator during patient follow-up and monitoring of disease progression from cirrhosis to liver cancer. To the best of our knowledge, however, the expression of LASP1 in OS and its effect on the proliferation and invasion of OS cells have not been reported.

The present study aimed to investigate the expression levels of H19 in OS and determine whether it regulates the expression of LASP1 by competitively binding to miR-29a-3p. The effect of H19 on OS was investigated by Cell Counting Kit (CCK)-8, western-blot, reverse transcription-quantitative (RT-q)PCR, wound healing and Transwell assays. The aim was to elucidate whether $\mathrm{H} 19$ is a novel competitive endogenous ncRNA involved in the proliferation, invasion and metastasis of OS cells, investigate the function of the H19/miR-29a-3p/LASP1 regulatory network in this process and determine whether targeting this pathway may provide novel approaches to the treatment of OS.

\section{Materials and methods}

Tissue samples. OS tissue samples [osteoblastic type by Dahlin standard (33)] and adjacent tissue (distance, 2-3 cm) were collected from six patients (four males and two females; mean age, $35.12 \pm 1.18$ years) with OS diagnosed at the First Affiliated Hospital of Harbin Medical University (Harbin, China) from June 2018 to June 2019 who had undergone surgery. Inclusion criteria were as follows: i) no other distant metastatic lesions; ii) OS diagnosed by preoperative puncture or postoperative pathology; iii) no history of other tumors; iv) no other disease affecting the treatment process and v) no preoperative treatment. Exclusion criteria were as follows: i) multiple lesions at the first diagnosis; ii) lesions located outside the limbs and iii) other diseases affecting the progression of treatment. Following collection, the six pairs of samples were immediately snap-frozen and stored at $-80^{\circ} \mathrm{C}$ for subsequent RNA extraction. The present study was approved by the Research Ethics Committee of Harbin Medical University and written informed consent was provided by all subjects.

Cell culture and transfection. The Cell Bank of Type Culture Collection of The Chinese Academy of Sciences provided all experimental cell lines used, including human osteoblasts (hFOB 1.19) and OS cells (MG-63 and Saos-2). The cells were cultured in Dulbecco's modified Eagle's medium (DMEM; HyClone; Cytiva) supplemented with $10 \%$ fetal bovine serum (Biological Industries; Sartorius AG) in a humidified environment at $37^{\circ} \mathrm{C}$ and $5 \% \mathrm{CO}_{2}$. miR-29a-3p mimic (50 $\left.\mathrm{nM}\right)$ and anti-miR-oligonucleotides (AMO)-miR-29a-3p inhibitor (100 nM; Table I) were transfected for $24 \mathrm{~h}$ at $37^{\circ} \mathrm{C}$ which were used to overexpress and inhibit expression of miR-29a-3p, and small interfering (si)RNA non-targeting sequence against human H19 (5'-CCTCTAGCTTGGAAATGAA-3'; $50 \mathrm{nM}$ ) were constructed by Guangzhou RiboBio Co., Ltd. and transfected for $24 \mathrm{~h}$ at $37^{\circ} \mathrm{C}$. The cells were transfected according to the manufacturer's instructions (X-tremeGENE siRNA Transfection Reagent; Roche Diagnostics).

$R N A$ extraction and $R T-q P C R$. Total RNA from tissues and cells was extracted using TRIzol ${ }^{\circledR}$ reagent (Invitrogen; Thermo Fisher Scientific, Inc.) and RNA concentration was estimated using a NanoDrop spectrophotometer (NanoDrop Technologies; Thermo Fisher Scientific, Inc.). The RT-qPCR primers were obtained from Invitrogen (Thermo Fisher Scientific, Inc.). cDNA was synthesized from total RNA and the relative mRNA and non-coding RNA levels were determined using a RT kit (Toyobo Life Science). The expression levels of miR-29a-3p were determined by normalization to U6 small nuclear RNA by the $2^{-\Delta \Delta C q}$ method (34). An ABI 7500 Sequence Detection System (Thermo Fisher Scientific, Inc.) and SYBR-Green Real-Time PCR Master Mix (Toyobo Life Science) were used to perform RT-qPCR according to the manufacturer's instructions. A total of $20 \mu \mathrm{l}$ PCR reaction mixture consisting of $1 \mu \mathrm{l}$ reverse primers, $1 \mu \mathrm{l}$ forward primers, $10 \mu \mathrm{l}$ SYBR-Green Master Mix, $6 \mu \mathrm{l}$ DEPC and $2 \mu 1$ synthesized cDNA was used. GAPDH was used as an internal control. RT was performed as follows: $25^{\circ} \mathrm{C}$ for $10 \mathrm{~min}, 37^{\circ} \mathrm{C}$ for $120 \mathrm{~min}, 85^{\circ} \mathrm{C}$ for $15 \mathrm{~min}$, then $4^{\circ} \mathrm{C}$. PCR conditions were as follows: $94^{\circ} \mathrm{C}$ for $5-10 \mathrm{~min}$, followed by 40 cycles of $95^{\circ} \mathrm{C}$ for $15 \mathrm{sec}, 60^{\circ} \mathrm{C}$ for $15 \mathrm{sec}$ and $72^{\circ} \mathrm{C}$ for $45 \mathrm{sec}$ and final extension at $72^{\circ} \mathrm{C}$ for 2-3 min. LASP1 expression levels were normalized to those of GAPDH using the $2^{-\Delta \Delta \mathrm{Cq}}$ method. The primer sequences are listed in Table I.

Western blot analysis. Following rinsing in ice-cold PBS, cells were lysed in radioimmunoprecipitation assay buffer containing $1 \%$ protease inhibitor (Thermo Fisher Scientific, Inc.), protein concentration was detected by NanoDrop. A total of $50 \mu \mathrm{g}$ protein per lane was separated using $10 \%$ SDS-PAGE. The proteins were transferred to nitrocellulose membranes (Thermo Fisher Scientific, Inc.), which were blocked in Tris-buffered saline containing $0.1 \%$ Tween-20 and 5\% non-fat milk (BD Biosciences) for $2 \mathrm{~h}$ at room temperature. The membranes were sequentially incubated with the appropriate primary antibodies against LASP1 (1:1,000; cat. no. ab156872; Abcam) overnight at $4{ }^{\circ} \mathrm{C}$ by gentle shaking and subsequently with Alexa Fluor ${ }^{\circledR}$ 647-conjugated goat anti-rabbit secondary antibodies (1:1,000; cat. no. 4414; Cell Signaling Technology, Inc.) for $1 \mathrm{~h}$ at room temperature. The Odyssey fluorescence scanning system (LI-COR Biosciences) and Image Studio software (V4.0; LI-COR Biosciences) were used to detect immunoreactivity. GAPDH (1:1,000; cat. no. ab9485; Abcam) was used as a loading control.

Cell proliferation assay. CCK-8 (Dojindo Molecular Technologies, Inc.) proliferation kit was used according to the manufacturer's instructions. Saos-2 and MG-63 cells were incubated for $24 \mathrm{~h}$ in 96 -well plates at a density of $1 \times 10^{4}$ cells/well. A total of $10 \mu \mathrm{lCCK}-8$ solution was added to the cells, which were incubated for $2 \mathrm{~h}$ at $37^{\circ} \mathrm{C}$. A microplate reader was used to evaluate the absorbance at $450 \mathrm{~nm}$. 
Table I. Primer sequences.

\begin{tabular}{lll}
\hline Name & \multicolumn{1}{c}{ Sequence, $5^{\prime} \rightarrow 3^{\prime}$} & Length, bp \\
\hline GAPDH & F: AAGAAGGTGGTGAAGCAGGC & 20 \\
& R: TCCACCACCCTGTTGCTGTA & 20 \\
LASP1 & F: CTGGAATGGGAGACCTGTTG & 20 \\
& R: CCCTGGATTGTGTGGGTATG & 20 \\
U6 & F: CTCGCTTCGGCAGCACATATACT & 23 \\
miR-29a-3p & R: ACGCTTCACGAATTTGCGTGTC & 22 \\
& F: AGCACCAUCUGAAAUCGGUUA & 21 \\
H19 & R: GTGCAGGGTCCGAGGT & 16 \\
miR-29a-3p mimic & F: ATCGGTGCCTCAGCGTTCGG & 20 \\
& R: CTGTCCTCGCCGTCACACCG & 20 \\
miR-29a-3p inhibitor & F: UAGCACCAUCUGAAAUCGGUUA & 22 \\
\hline
\end{tabular}

LASP1, LIM and SH3 domain protein 1; miR, microRNA; F, forward; R, reverse.

Wound healing assay. OS cells were cultured in six-well plates to $80-90 \%$ confluence, serum-starved for $6 \mathrm{~h}$ and siRNA was transfected into the cells as aforementioned. Following $24 \mathrm{~h}$ incubation at $37^{\circ} \mathrm{C}$, a wound was made on all plate surfaces using a $200-\mu 1$ sterile plastic pipette tip, the plates were washed with PBS and images were obtained by light microscopy after $24 \mathrm{~h}$ (magnification, x100). All tests were performed in triplicate and the experiments were repeated at least two times.

Transwell assay. Transwell filters (pore size, $8 \mu \mathrm{m}$; BD Biosciences) were added to 24 -well plates. Following transfection, Saos-2 and MG-63 cells were incubated at $37^{\circ} \mathrm{C}$ for $24 \mathrm{~h}$ and resuspended in DMEM (HyClone; Cytiva) with 5\% fetal bovine serum (Biological Industries; Sartorius AG). The concentration was adjusted to $2.5 \times 10^{5}$ cells $/ \mathrm{ml}$. Following an additional culture for $24 \mathrm{~h}$ at $37^{\circ} \mathrm{C}$, the cells and the Matrigel on the upper side of the membrane were gently removed with clean cotton swabs, while cells on the bottom of the membrane surface were fixed with $100 \%$ cold methanol for $30 \mathrm{~min}$ at room temperature. Following fixation, the cells were stained with $0.1 \%$ crystal violet for $10 \mathrm{~min}$ at room temperature. Cell invasion was quantified based on the average number of cells in each of the three inserts by microscopy (Olympus BX53; magnification, $\mathrm{x} 400$ ).

Xenograft mouse model. All experiments involving animals were approved by the Ethics Committee of Harbin Medical University. Nude mice (age, 4 weeks; weight, $16 \mathrm{~g}$; Weitong Lihua) were housed at $18-25^{\circ} \mathrm{C}$ and $50-60 \%$ relative humidity with a 12/12-h light/dark cycle and access to sufficient food and water. The mice were anesthetized by intraperitoneal injection of $1 \%$ sodium pentobarbital at a dose of $50 \mathrm{mg} / \mathrm{kg}$. MG-63 $\left(1 \times 10^{7}\right)$ and Saos-2 cells $\left(7 \times 10^{6}\right)$ were transfected with H19 siRNA or empty vector plasmid (Guangzhou RiboBio Co., Ltd.). A total of 18 female athymic nude mice were divided into six groups (n=3/group) as follows: MG-63,
MG-63 + siH19, MG-63 + si negative control (NC), Saos-2, Saos- $2+$ siH19 and Saos- $2+$ siNC. The cells were cultured for $48 \mathrm{~h}$ and resuspended in $100 \mu \mathrm{l}$ serum-free DMEM (HyClone; Cytiva) at $37^{\circ} \mathrm{C}$. Subsequently, the cells were subcutaneously injected on the back of mice. The tumor xenografts were grown for 3 weeks. The tumors were removed and the diameters were determined. The mice were sacrificed by cervical dislocation.

Prediction of the regulatory association between H19, miR-29a-3p and LASP1. The association between H19, miR-29a-3p and LASP1 was examined using the bioinformatics tool starBase v2.0 (35).

Statistical analysis. The data are expressed as the mean \pm SEM and were analyzed using SPSS 18.0 software (SPSS, Inc.). Each experiment was repeated at least three times. Mann-Whitney $\mathrm{U}$ test and paired t-test were used to compare differences between two experimental groups, while one-way ANOVA with post hoc Student-Newman-Keuls and Tukey's tests were used to compare differences among multiple experimental groups. $\mathrm{P}<0.05$ (two-tailed) was considered to indicate a statistically significant difference.

\section{Results}

LASP1 and H19 are involved in OS progression. Expression levels of LASP1 were investigated in OS and normal adjacent tissue using RT-qPCR and western blot analysis. The expression levels of LASP1 were higher in OS tissue compared with normal tissue (Fig. 1A and B). LASP1 expression was also assessed in MG-63, Saos-2 and hFOB 1.19 cells; LASP1 levels were lower in hFOB 1.19 cells than in MG-63 and Saos-2 OS cells (Fig. 1C and D). Subsequently, the expression levels of lncRNA H19 were evaluated in OS tissue and cell lines; OS exhibited higher levels of H19 compared with normal tissue (Fig. 1E). Similarly, H19 expression levels were lower in hFOB 1.19 cells compared with in MG-63 and Saos-2 cells (Fig. 1F). 
A
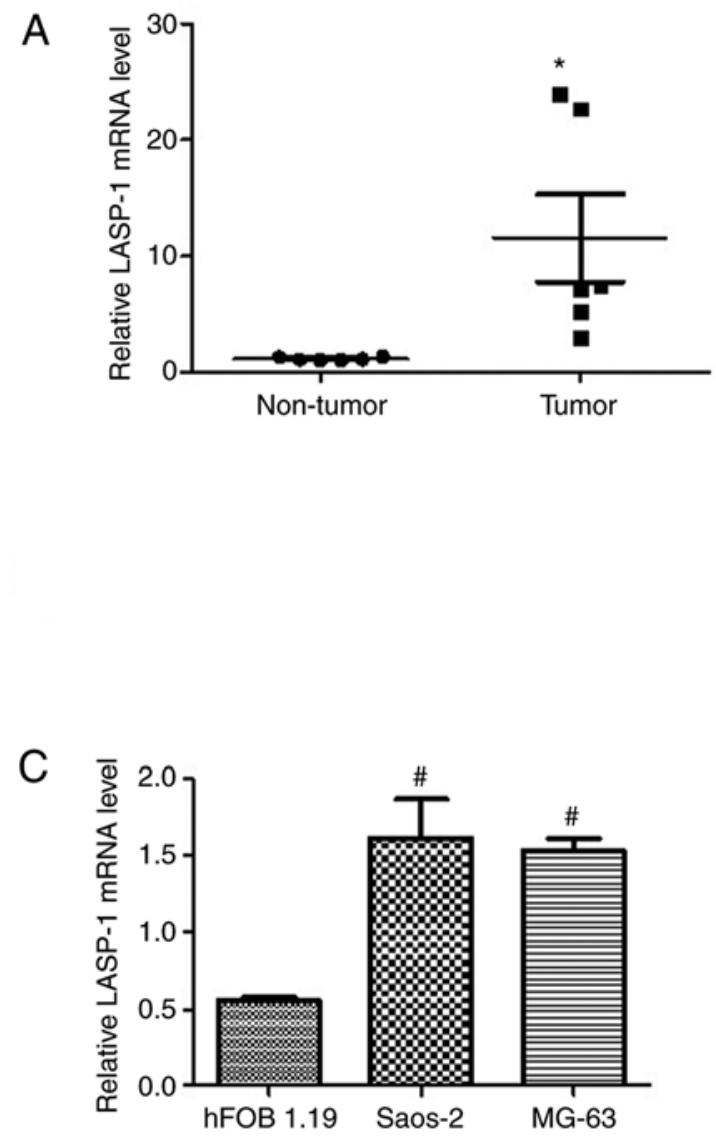

E

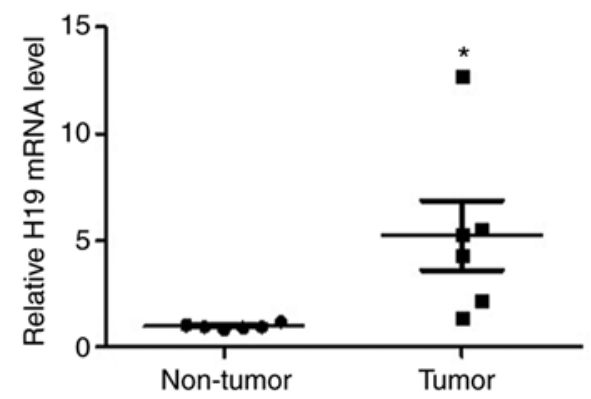

B
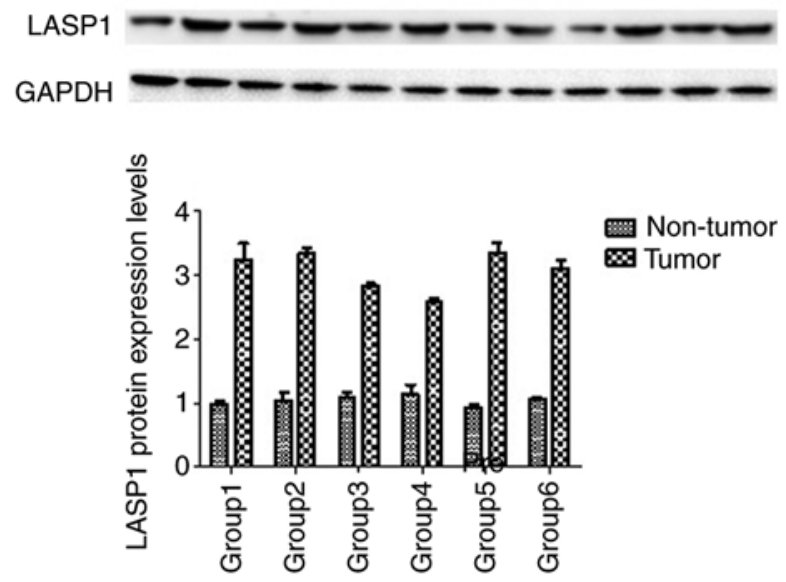

D

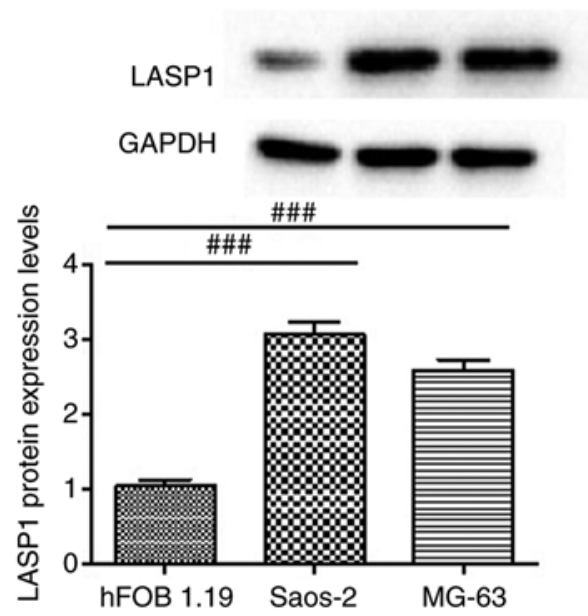

F

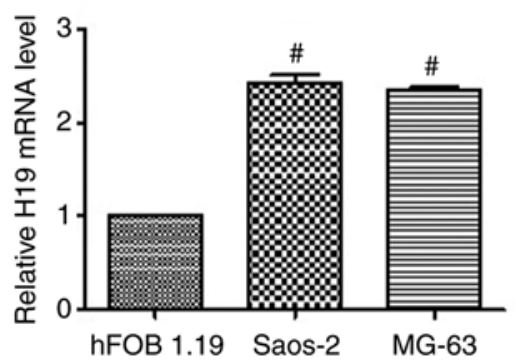

Figure 1. Expression levels of LASP1 and H19 are elevated in osteosarcoma. (A) mRNA and (B) protein expression levels of LASP1 were significantly higher in tumor compared with non-tumor tissue and lower in hFOB 1.19 cells than in MG63 or Saos-2 cells, as shown by (C) RT-qPCR and (D) western blotting. (E) mRNA expression levels of H19 were significantly higher in tumor compared with non-tumor tissue and (F) lower in hFOB 1.19 cells than MG63 or Saos-2 cells, as shown by RT-qPCR. Data are expressed as the mean \pm SEM $(\mathrm{n}=3)$. ${ }^{*} \mathrm{P}<0.05$ vs. non-tumor; ${ }^{\#} \mathrm{P}<0.05$, ${ }^{\# \#} \mathrm{P}<0.001 \mathrm{vs}$. hFOB 1.19 . LASP1, LIM and SH3 domain protein 1; RT-q, reverse transcription-quantitative.

These findings suggested that expression levels of H19 and LASP1 were associated with OS formation.

Proliferation and invasion of $O S$ cells are inhibited by H19 knockdown. The effect of H19 on OS cell invasion and proliferation was investigated. Successful H19 knockdown was performed by siRNA (Fig. 2A) and Transwell invasion, wound healing and CCK-8 assays were used to measure OS cell invasion, migration and proliferation, respectively. CCK-8 assay indicated that proliferation of Saos-2 and MG-63 cells was inhibited by H19 knockdown compared with control cells (Fig. 2B). In addition, H19 knockdown inhibited Saos-2 and MG-63 cell migration and invasion compared with that of the control group (Fig. 2C and D). Overall, the results demonstrated that $\mathrm{H} 19$ promoted OS cell invasion and proliferation in vitro.

miR-29a-3p is a potential factor regulating the H19/LASPI pathway. The aforementioned experiments indicated an association between expression levels of H19 and LASP-1 in both OS and adjacent normal tissue. To assess this, miRs that serve an important regulatory role between H19 and LASP1 were searched. The potential complementary miR and H19 base pairs were examined using the bioinformatics tool starBase 
A

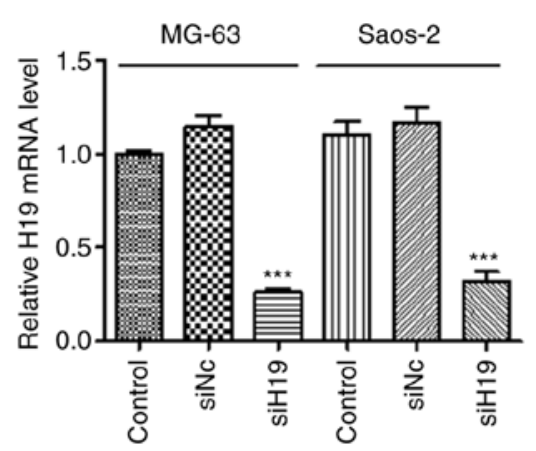

B

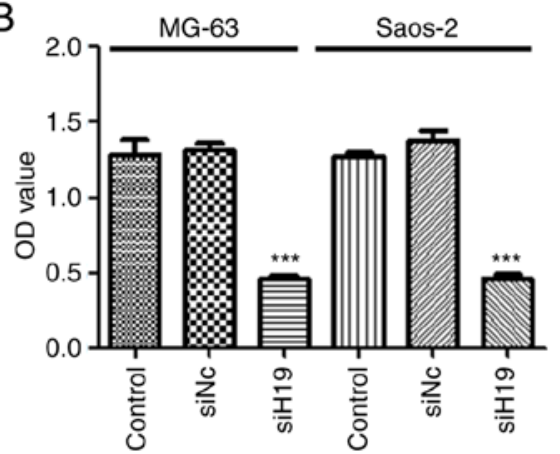

C
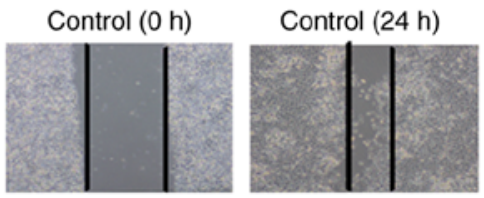

$\operatorname{siNc}(0 \mathrm{~h})$
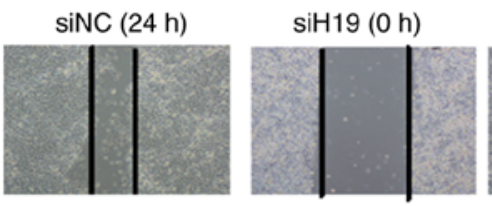

$\operatorname{siH} 19(24 \mathrm{~h})$

MG-63
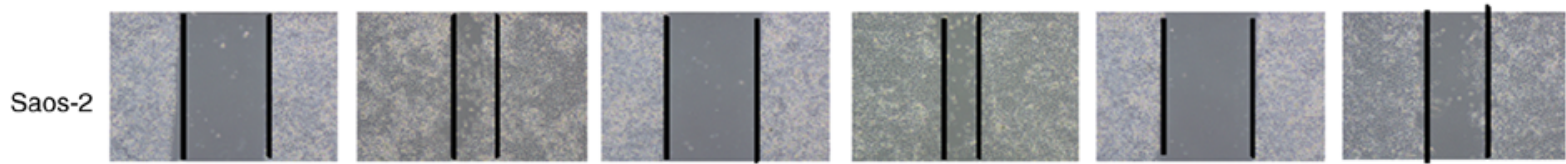

MG-63
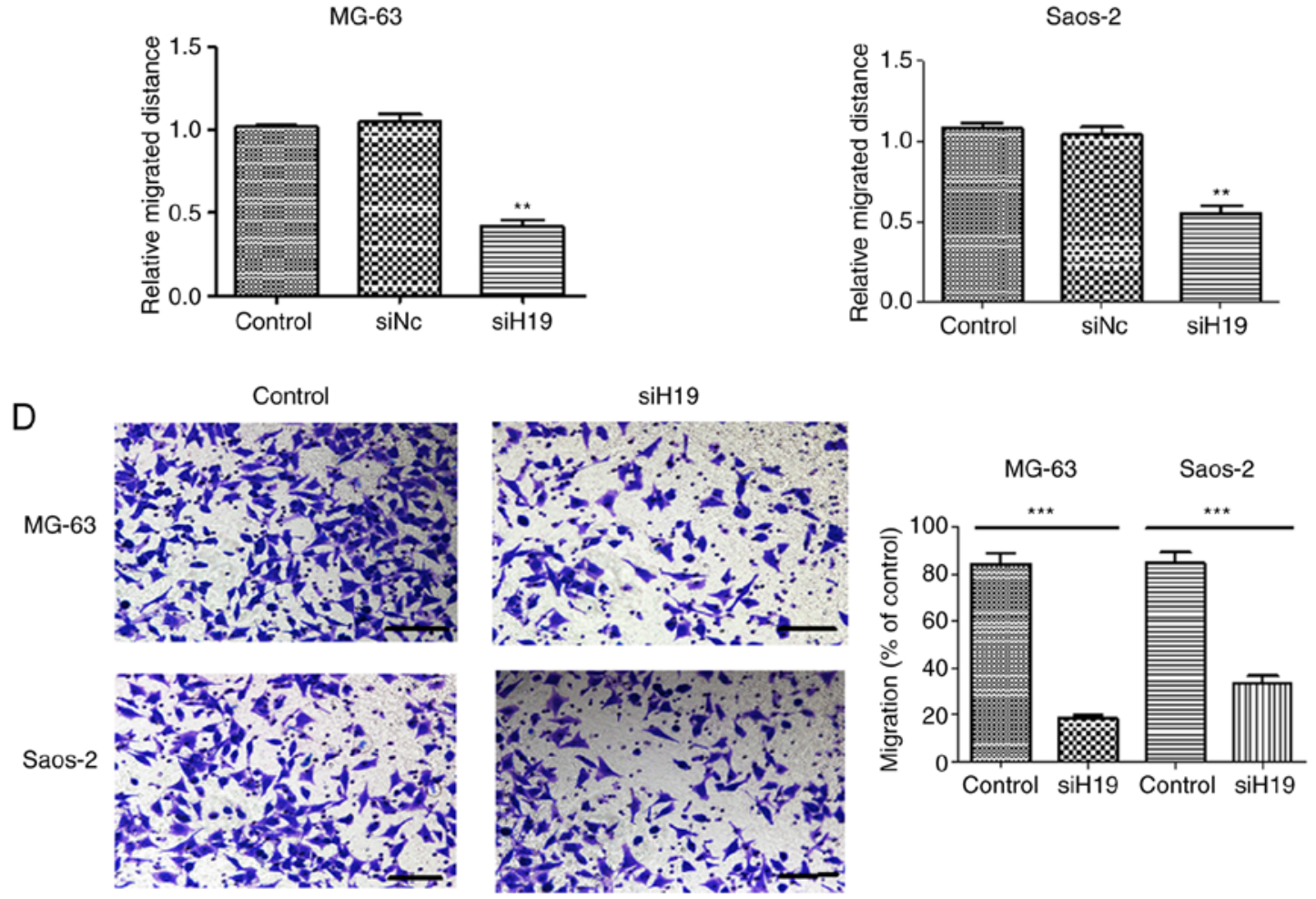

Figure 2. H19 siRNA inhibits proliferation, migration and invasion of osteosarcoma cells. (A) H19 siRNA was successfully transfected into MG-63 and Saos-2 cells. (B) H19 siRNA decreased the proliferative capacity of MG-63 and Saos-2 cells, as shown by Cell Counting Kit-8 assay. OD values (450 nm) were quantitated. H19 siRNA decreased (C) migration and (D) invasion of MG-63 and Saos-2 cells vs. control and siNC groups. Magnification, x100. Scale bar, $50 \mu \mathrm{m}$. Data are expressed as the mean $\pm \mathrm{SEM}(\mathrm{n}=3) .{ }^{* *} \mathrm{P}<0.01$ and ${ }^{* * *} \mathrm{P}<0.001$ vs. control. si, small interfering; OD, optical density; NC, negative control.

v2.0, which revealed that H19 and LASP1 shared a common target, miR-29a-3p (Fig. 3A). Jia et al (36) previously reported that miR-29a-3p targets H19 in glioma-associated endothelial cells, while a study of lung cancer tissue demonstrated that LASP1 expression is inhibited by miR-29a-3p (37). To determine the association between the onset of OS and expression levels of miR-29a-3p, the latter were detected by both in vivo and in vitro assays. The expression levels of miR-29a-3p were higher in adjacent normal compared with OS tissue, Saos-2 and MG63 cells (Fig. 3B and C).

siNC or H19 siRNA were transfected into Saos-2 and MG-63 cells and expression levels of miR-29a-3p were assessed to determine whether H19 targeted miR-29a-3p. miR-29a-3p expression was increased in both MG-63 and Saos- 2 cells 

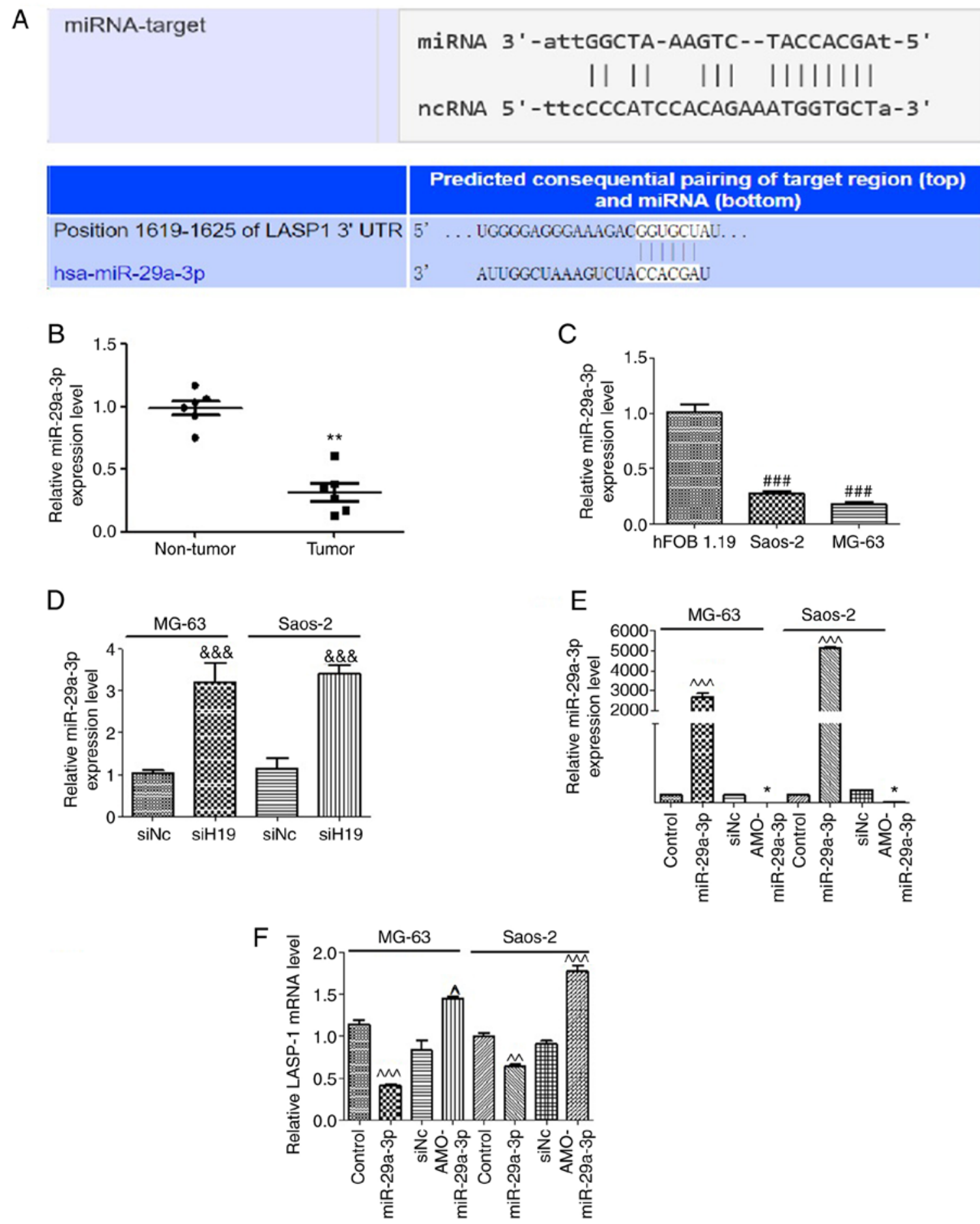

Figure 3. miR-29a-3p is a potential factor in the H19-LASP1 pathway. (A) Bioinformatics software (starbase V2.0) prediction of biological targets common to H19 and LASP1. (B) mRNA expression levels of miR-29a-3p were significantly lower in six pairs of osteosarcoma tissue than in corresponding adjacent tissue, as shown by RT-qPCR. (C) miR-29a-3p levels were higher in hFOB 1.19 cells than MG63 or Saos-2 cells. (D) Expression of miR-29a-3p was increased following transfection of H19 siRNA into MG63 or Saos-2 cells. (E) miR-29a-3p mimic and inhibitor were successfully transfected in MG-63 and Saos-2 cells. (F) Relative expression of LASP1 in MG63 or Sao-2 cells following transfection with miR-29a-3p mimic and inhibitor, as determined by RT-qPCR. Data are expressed as the mean $\pm \mathrm{SEM}(\mathrm{n}=3) .{ }^{*} \mathrm{P}<0.05,{ }^{* *} \mathrm{P}<0.01$ vs. non-tumor; ${ }^{\# \# \#} \mathrm{P}<0.001$ vs. $\mathrm{hFOB} 1.19 ;{ }^{\text {\&\&\& }} \mathrm{P}<0.001$ vs. siNC; ${ }^{\wedge} \mathrm{P}<0.05,{ }^{\wedge} \mathrm{P}<0.01$ and ${ }^{\wedge \wedge} \mathrm{P}<0.001$ vs. control. miR, microRNA; LASP1, LIM and SH3 domain protein 1; RT-q, reverse transcription-quantitative; nc, non-coding; UTR, untranslated region; si, small interfering; NC, negative control; AMO, anti-miR-oligonucleotides.

following transfection with H19-siRNA, as determined by RT-qPCR analysis (Fig. 3D).

To verify whether LASP1 is a downstream target of miR-29a-3p, the effects of overexpression of this miR on LASP1 expression were investigated (Fig. 3E). AMO-miR-29a-3p
(miR-29a-3p), siNC, miR-29a-3p mimic and control sequences were transfected into Saos- 2 and MG-63 cells. The expression levels of LASP1 were decreased following overexpression of miR-29a-3p and the opposite results were noted in miR-29a-3p inhibitor transfected cells (Fig. 3F). These results suggested 

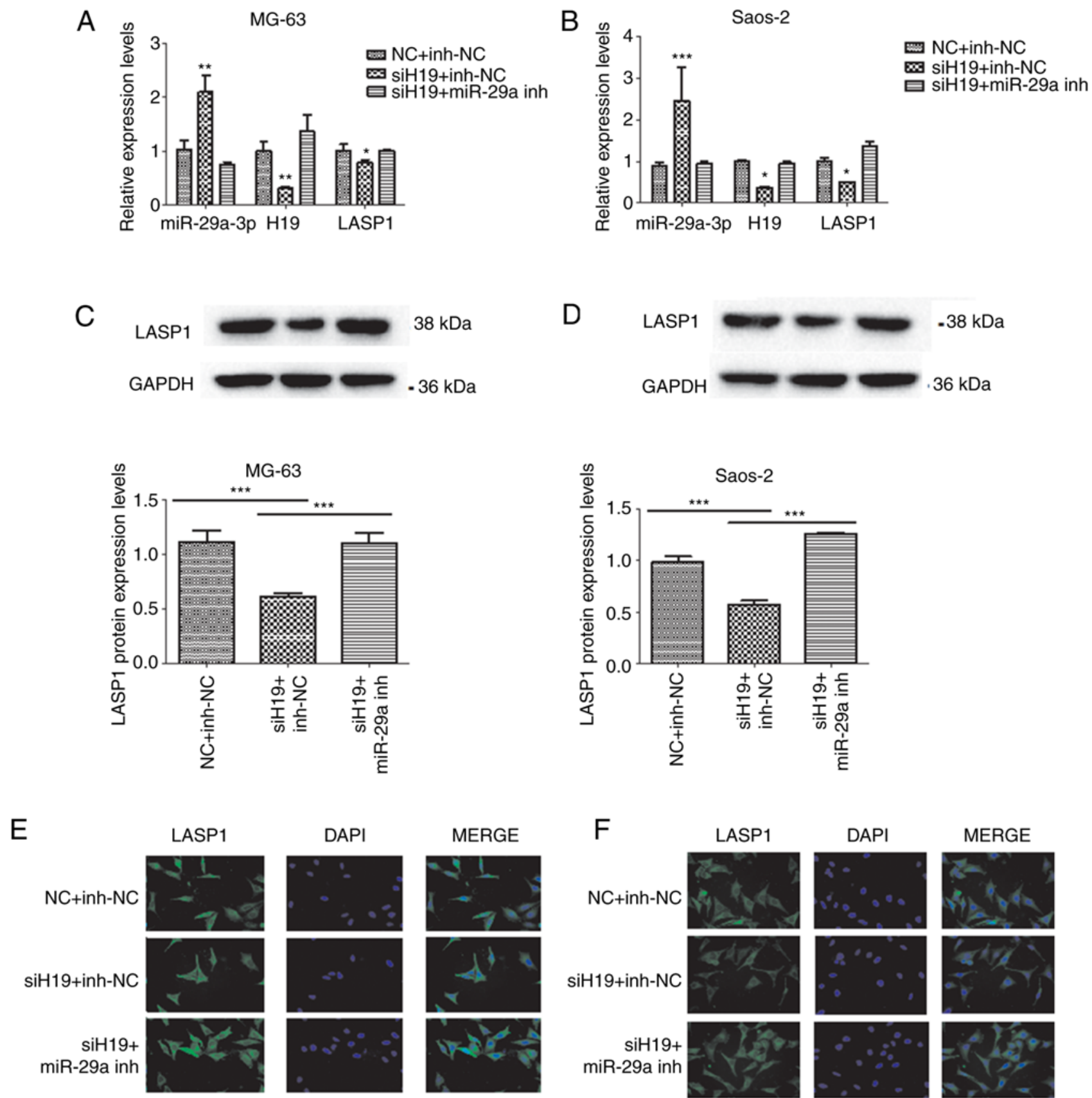

MG-63
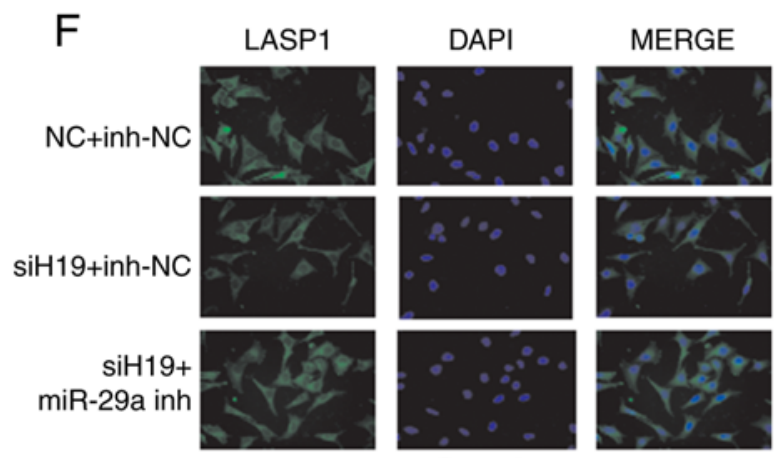

Figure 4. miR-29a-3p reverses the effects of H19 on OS cells. miR-29a-3p expression was elevated in (A) MG-63 and (B) Saos-2 cells transfected with H19 siRNA. LASP1 mRNA and protein expression levels were decreased by inhibition of H19 and restored by miR-29a-3p inh in (C and E) MG-63 and (D and F) Saos-2 cells. Data are expressed as the mean \pm SEM $(n=3) .{ }^{*} \mathrm{P}<0.05,{ }^{* * *} \mathrm{P}<0.01$ and ${ }^{* * * *} \mathrm{P}<0.001 \mathrm{vs}$. NC + inh-NC. miR, microRNA; si, small interfering; LASP1, LIM and SH3 domain protein 1; inh, inhibitor; NC, negative control.

that H19 affected LASP1 expression by competitively binding to miR-29a-3p.

miR-29a-3p reverses the effects of $H 19$ on OS cells. Although the aforementioned analysis demonstrated that H19 targeted miR-29a-3p, the precise mechanism of its action in OS cells remains unclear. Additional experiments were performed to assess the effects of H19 on OS via the H19/miR-29a-3p/LASP1 axis. miR-29a-3p inhibitor reversed the inhibitory effect of H19 siRNA on LASP1 expression, as determined by western blotting and RT-qPCR (Fig. 4A-C). Furthermore, the effects of H19 on OS cells were reversed by miR-29a-3p, whereas
H19 increased OS cell proliferation and invasion via the H19/miR-29a-3p/LASP1 axis.

H19 promotes proliferation of OS. To confirm whether H19 affects the proliferative capacity of OS cells, H19 siRNA and lentiviral vectors were transfected into Saos-2 and MG-63 cells. These cells were subcutaneously injected into female athymic nude mice and spontaneously grown for 3 weeks prior to tumor isolation. The body weight of mice was measured and recorded at 0, 2 and 3 weeks (Fig. 5A and D). At 2 weeks, the mice gained weight because of the small size of the tumor, but the body weight of mice in each group decreased at 3 weeks 
A

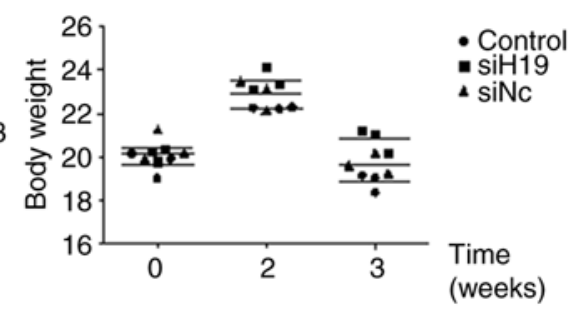

D

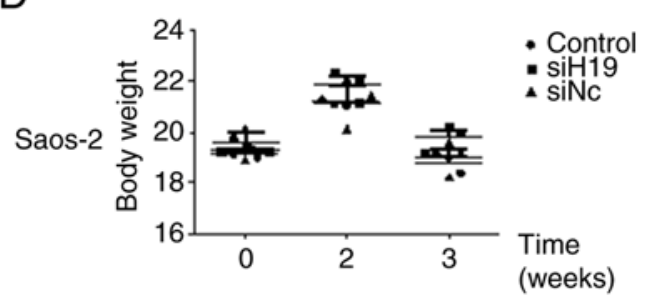

$\mathrm{B}$
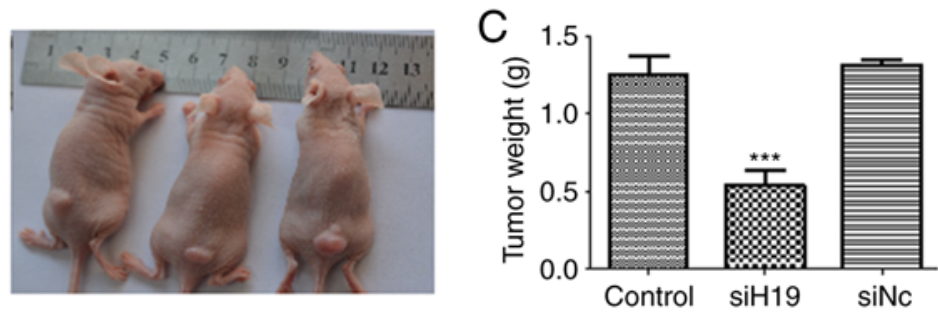

$\mathrm{E}$

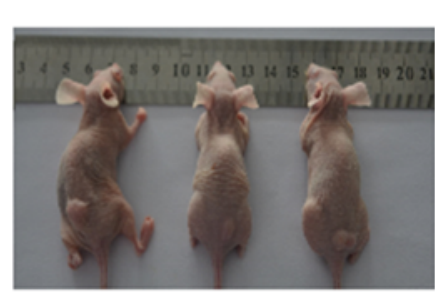

$\mathrm{F}$

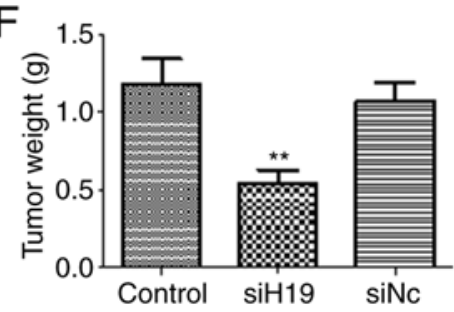

Figure 5. H19 promotes proliferation of osteosarcoma cells. Injected cells were transfected with H19 siRNA or siNC. (A) Body weight and tumor (B) growth and (C) weight of mice injected with MG-63 cells. (D) Body weight and tumor (E) growth and (F) weight of mice injected with and Saos-2 cells. ** P $<0.01$ and ${ }^{* * * *} \mathrm{P}<0.001$ vs. control. si, small interfering; $\mathrm{NC}$, negative control.

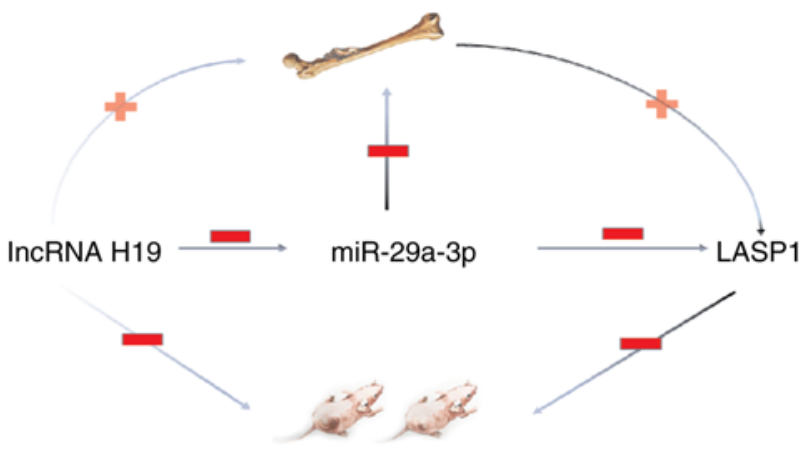

Figure 6. Model of H19-miR-29a-3p-LASP1 regulation in OS. +, promotion; -, suppression. LASP1 may be associated with the onset of OS. H19 inhibits miR-29a-3p expression by directly interacting with miR-29a-3p and indirectly affecting expression of the downstream miR-29a-3p target LASP1. miR, microRNA; LASP1, LIM and SH3 domain protein 1; OS, osteosarcoma.

because the increase in tumor volume affected growth of nude mice. The mice were anesthetized and dorsal tumor images were captured. The maximum tumor diameter in the present study was $0.9 \mathrm{~cm}$ and the maximum tumor volume was $0.074 \mathrm{~cm}^{3}$. By contrast, tumor growth in the H19 knockdown group was significantly inhibited compared with that in the control group, suggesting that H19 may be involved in the onset and progression of OS and affect the proliferative capacity of OS cells (Fig. 5B, C, E and F).

\section{Discussion}

OS is a malignant tumor that occurs in adolescents and is characterized by insidious onset and distant metastasis at the early stage of disease (1). Adjuvant chemotherapy and surgical treatment are the most commonly used methods for treatment of OS (18). However, the results have not been not encouraging (38). IncRNAs are a class of heterogeneous molecules that mediate gene expression by regulating transcription, protein and miR function $(7,8)$. It has been shown that $\mathrm{H} 19$ is upregulated in OS (13). However, the functional role of H19 remains unknown. In the present study, expression levels of H19 were assessed in clinical tissue specimens and cell lines. Subsequent functional experiments indicated that H19 increased the proliferation, migration and invasion of OS cells by competitively binding to miR-29a-3p and affecting transcription of LASP1.

Numerous studies have investigated the function of IncRNAs to elucidate their mechanisms of action in the development of a number of diseases $(39,40)$. Certain studies have demonstrated that IncRNA participates in mRNA regulation by competitively binding with miRNA $(41,42)$. To the best of our knowledge, the present study is the first to demonstrate the effect of LASP1 on OS. The present experiments indicated significantly higher levels of LASP1 in OS than in adjacent normal tissue. LASP1 is a member of the nebulin family and is characterized by a LIM motif (cysteine-rich LIM/double zinc finger motif) (28). It exhibits structural characteristics that include the LIM motif and proto-oncogene tyrosine-protein kinase Src homology region 3 (43). LASP1 dysregulation has been noted in specific tumors, such as breast and non-small cell lung cancer $(27,31)$. In addition, LASP1 promotes the invasion and proliferation of tumor cells and high LASP1 expression is associated with poor prognosis in patients with gastric cancer (44).

The present study provided evidence to support the tumorigenic role of LASP1 in OS tissue. The data demonstrated the significant effects of LASP1 on the onset and progression of OS. Nonetheless, the involvement of LASP1 in the pathogenesis and progression of OS has not been fully elucidated.

The expression levels of miR-29a-3p were measured by in vivo and in vitro assays. The induction of epithelial-to-mesenchymal transition (EMT) has been shown to serve a key role in the metastasis of tumor cells. High expression levels of miR-29a induce EMT in various types of tumor cell (45). Although EMT has been shown to regulate the invasion and 
metastasis of several types of cancer, including OS, a limited number of studies have investigated the expression levels of miR-29a-3p in patients with OS $(46,47)$. The present study did not fully elucidate the biological mechanism of miR-29a-3p in OS. RT-qPCR demonstrated higher miR-29-3p levels in adjacent non-tumor tissue than in OS tissue. In addition, the OS cell lines MG-63 and Saos-2 exhibited significantly lower miR-29a-3p expression compared with that observed in hFOB 1.19 cells.

Saos-2 and MG-63 cells were transfected with AMO-miR-29a-3p, siNC, miR-29a-3p mimic and control sequences to assess the association between LASP1 and miR-29a-3p. These experiments revealed that LASP1 expression was decreased in miR-29a-3p-overexpressing cells, while the opposite result was noted in cells expressing AMO-miR-29a-3p. The findings of the present study indicated that LASP1 was a downstream target of miR-29a-3p and that both LASP1 and miR-29a-3p served a key role in the development of OS.

lncRNA H19 is a paternally imprinted gene located on chromosome 11p11.5 (48). The genetic locus of lncRNA H19 is adjacent to the telomeric region, which participates in the development and progression of tumors in adults as well as children (49). Li et al (13) suggested that $\mathrm{H} 19$ acted as a competing endogenous RNA that decreased miR-200 activity in OS and increased the risk of cancer metastasis. Chan et al (50) demonstrated that $\mathrm{H} 19$ overexpression induces OS development. The aforementioned findings confirmed that H19 was associated with the incidence of OS. The present study indicated that H19 overexpression served an important role in OS tissue. H19 was also shown to be a target of miR-29a-3p, while both H19 and miR-29a-3p levels were suppressed. Therefore, the data demonstrated that $\mathrm{H} 19$ served as an endogenous miR sponge that bound to miR-29a-3p and regulated its function. In addition, expression levels of H19 and miR-29a-3p exhibited opposite trends in OS, suggesting a potential association between these molecules with regard to regulation of OS development.

The results of the present study indicated that H19 may promote proliferation and invasion of OS via the miR-29a-3p/LASP1 pathway. It was also demonstrated that LASP1 may be associated with the onset of OS, whereas H19 inhibited miR-29a-3p expression by directly interacting with miR-29a-3p and indirectly affecting expression of the downstream miR-29a-3p target LASP1 (Fig. 6). Therefore, targeting this pathway may provide novel therapeutic strategies for the treatment of OS. However, due to the small sample size of OS, the present study has certain limitations. Expanding the sample size will confirm that H19-miR-29a-3p is an important regulatory mechanism in OS.

\section{Acknowledgements}

Not applicable.

\section{Funding}

The present study was supported by Research Innovation Fund of the First Affiliated Hospital of Harbin Medical University (grant nos. 2019B04 and 2019B12), The Heilongjiang Provincial Natural Science Foundation (grant no. H2017027),
The Shandong Provincial Natural Science Foundation (grant no. ZR2014HP027) and The Linyi Municipal Science and Technology Development Plan (grant no. 201818015).

\section{Availability of data and materials}

The datasets used and/or analyzed during the current study are available from the corresponding author on reasonable request.

\section{Authors' contributions}

$\mathrm{XJ}$ and WW confirm the authenticity of all the raw data. $\mathrm{HJ}$ designed and performed the experiments. XJ and HW performed the experiments. WW analyzed data. HJ wrote the manuscript. All authors read and approved the final manuscript.

\section{Ethics approval and consent to participate}

The present study was approved by The First Affiliated Hospital of Harbin Medical University and all patients provided written informed consent.

\section{Patient consent for publication}

Not applicable.

\section{Competing interests}

The authors declare that they have no competing interests.

\section{References}

1. Kansara M, Teng MW, Smyth MJ and Thomas DM: Translational biology of osteosarcoma. Nat Rev Cancer 14: 722-735, 2014.

2. Yamamoto Y, Kanzaki R, Kanou T, Ose N, Funaki S, Shintani Y, Minami M, Outani H, Takenaka S, Hamada K, et al: Long-term outcomes and prognostic factors of pulmonary metastasectomy for osteosarcoma and soft tissue sarcoma. Int J Clin Oncol 24: 863-870, 2019.

3. Bishop MW, Janeway KA and Gorlick R: Future directions in the treatment of osteosarcoma. Curr Opin Pediatr 28: 26-33, 2016.

4. Gao K, Su Z, Liu H and Liu Y: Anti-proliferation and anti-metastatic effects of sevoflurane on human osteosarcoma U2OS and Saos-2 cells. Exp Mol Pathol 108: 121-130, 2019.

5. Xu J, Ding R and Xu Y: Effects of long non-coding RNA SPRY4-IT1 on osteosarcoma cell biological behavior. Am J Transl Res 8: 5330-5337, 2016.

6. Kotake Y, Goto T, Naemura M, Inoue Y, Okamoto H and Tahara K: Long noncoding RNA PANDA positively regulates proliferation of osteosarcoma cells. Anticancer Res 37: 81-85, 2017.

7. Wen JF, Jiang YQ, Li C, Dai XK, Wu T and Yin WZ: LncRNA-SARCC sensitizes osteosarcoma to cisplatin through the miR-143-mediated glycolysis inhibition by targeting Hexokinase 2. Cancer Biomark 28: 231-246, 2020.

8. Fu D, Lu C, Qu X, Li P, Chen K, Shan L and Zhu X: LncRNA TTN-AS1 regulates osteosarcoma cell apoptosis and drug resistance via the miR-134-5p/MBTD1 axis. Aging (Albany NY) 11: 8374-8385, 2019.

9. Zhang YH, Fu J,Zhang ZJ, Ge CC and Yi Y: LncRNA-LINC00152 down-regulated by miR-376c-3p restricts viability and promotes apoptosis of colorectal cancer cells. Am J Transl Res 8: 5286-5297, 2016

10. Barbáchano A, Fernández-Barral A, Pereira F, Segura MF, Ordóñez-Morán P, Carrillo-de Santa Pau E, González-Sancho JM, Hanniford D, Martínez N, Costales-Carrera A, et al: SPROUTY-2 represses the epithelial phenotype of colon carcinoma cells via upregulation of ZEB1 mediated by ETS1 and miR-200/miR-150. Oncogene 35: 2991-3003, 2016. 
11. Kou JT, Ma J, Zhu JQ, Xu WL, Liu Z, Zhang XX, Xu JM, Li H, $\mathrm{Li} \mathrm{XL}$ and He Q: LncRNA NEAT1 regulates proliferation, apoptosis and invasion of liver cancer. Eur Rev Med Pharmacol Sci 24: 4152-4160, 2020.

12. Zhang X, Yao J, Shi H, Gao B and Zhang L: LncRNA TINCR/microRNA-107/CD36 regulates cell proliferation and apoptosis in colorectal cancer via PPAR signaling pathway based on bioinformatics analysis. Biol Chem 400: 663-675, 2019.

13. Li M, Chen H, Zhao Y, Gao S and Cheng C: H19 functions as a ceRNA in promoting metastasis through decreasing miR-200s activity in osteosarcoma. DNA Cell Biol 35: 235-240, 2016.

14. Li S, Pei Y, Wang W, Liu F, Zheng K and Zhang X: Circular RNA 0001785 regulates the pathogenesis of osteosarcoma as a ceRNA by sponging miR-1200 to upregulate HOXB2. Cell Cycle 18: 1281-1291, 2019 .

15. Pu M, Chen J, Tao Z, Miao L, Qi X, Wang Y and Ren J: Regulatory network of miRNA on its target: Coordination between transcriptional and post-transcriptional regulation of gene expression. Cell Mol Life Sci 76: 441-451, 2019.

16. Liu B, Li J and Cairns MJ: Identifying miRNAs, targets and functions. Brief Bioinform 15: 1-19, 2014

17. Liu H, Su P, Zhi L and Zhao K: miR-34c-3p acts as a tumor suppressor gene in osteosarcoma by targeting MARCKS. Mol Med Rep 15: 1204-1210, 2017

18. Zhang M, Wang D, Zhu T and Yin R: miR-214-5p targets ROCK1 and suppresses proliferation and invasion of human osteosarcoma cells. Oncol Res 25: 75-81, 2017.

19. Zhao B, Song X and Guan H: CircACAP2 promotes breast cancer proliferation and metastasis by targeting miR-29a/b-3p-COL5A1 axis. Life Sci 244: 117179, 2020.

20. Wang X, Liu S, Cao L, Zhang T, Yue D, Wang L, Ping Y, He Q, Zhang C, Wang M, et al: miR-29a-3p suppresses cell proliferation and migration by downregulating IGF1R in hepatocellular carcinoma. Oncotarget 8: 86592-86603, 2017.

21. Gado MM, Mousa NO, Badawy MA, El Taweel MA and Osman A: Assessment of the diagnostic potential of miR-29a-3p and miR-92a-3p as circulatory biomarkers in acute myeloid leukemia. Asian Pac J Cancer Prev 20: 3625-3633, 2019.

22. Cai J, Qiao B, Gao N, Lin N and He W: Oral squamous cell carcinoma-derived exosomes promote $\mathrm{M} 2$ subtype macrophage polarization mediated by exosome-enclosed miR-29a-3p. Am J Physiol Cell Physiol 316: C731-C740, 2019.

23. Liang J, Wang S and Wang Z: Role of microRNAs in embryo implantation. Reprod Biol Endocrinol 15: 90, 2017.

24. Sui Y, Zhang X, Yang H, Wei W and Wang M: MicroRNA-133a acts as a tumour suppressor in breast cancer through targeting LASP1. Oncol Rep 39: 473-482, 2018.

25. Ma X, Zhang H, Yin H, Geng S, Liu Y, Liu C, Zhao J, Liu Y, Wang X and Wang Y: Up-regulated microRNA-218-5p ameliorates the damage of dopaminergic neurons in rats with Parkinson's disease via suppression of LASP1. Brain Res Bull 166: 92-101, 2021.

26. Huang Z, Pang G, Huang YG and Li C: miR-133 inhibits proliferation and promotes apoptosis by targeting LASP1 in lupus nephritis. Exp Mol Pathol 114: 104384, 2020.

27. Zhang X, Liu Y, Fan C, Wang L, Li A, Zhou H, Cai L, Miao Y, Li Q, Qiu X and Wang E: Laspl promotes malignant phenotype of non-small-cell lung cancer via inducing phosphorylation of FAK-AKT pathway. Oncotarget 8: 75102-75113, 2017.

28. Yan P, Liu J, Zhou R, Lin C, Wu K, Yang S, Yang S, Zhou J, Xu L, Wang $\mathrm{H}$ and Zhao L: LASP1 interacts with $\mathrm{N}$-WASP to activate the Arp $2 / 3$ complex and facilitate colorectal cancer metastasis by increasing tumour budding and worsening the pattern of invasion. Oncogene 39: 5743-5755, 2020.

29. Dimova I, Orsetti B, Negre V, Rouge C, Ursule L, Lasorsa L, Dimitrov R, Doganov N, Toncheva D and Theillet C: Genomic markers for ovarian cancer at chromosomes 1,8 and 17 revealed by array CGH analysis. Tumori 95: 357-366, 2009.

30. Li Z, Chen Y, Wang X, Zhang H, Zhang Y, Gao Y, Weng M, Wang L, Liang H, Li M, et al: LASP-1 induces proliferation, metastasis and cell cycle arrest at the G2/M phase in gallbladder cancer by down-regulating S100P via the PI3K/AKT pathway. Cancer Lett 372: 239-250, 2016.

31. Shen Y, Yang Y and Li Y: MiR-133a acts as a tumor suppressor in lung cancer progression by regulating the LASP1 and TGF- $\beta / \mathrm{Smad} 3$ signaling pathway. Thorac Cancer 11: 3473-3481, 2020.
32. Yin L, Chen Y, Zhou Y, Deng G, Han Y, Guo C, Li Y, Zeng S and Shen H: Increased long noncoding RNA LASP1-AS is critical for hepatocellular carcinoma tumorigenesis via upregulating LASP1. J Cell Physiol 234: 13493-13509, 2019.

33. Meister P, Konrad E, Lob G, Janka G, Keyl W and Stürz H: Osteosarcoma: Histological evaluation and grading. Arch Orthop Trauma Surg 94: 91-98, 1979.

34. Livak KJ and Schmittgen TD: Analysis of relative gene expression data using real-time quantitative PCR and the 2(-Delta Delta C(T)) method. Methods 25: 402-408, 2001.

35. Du Y, Wei N, Hong J and Pan W: Long non-coding RNASNHG17 promotes the progression of breast cancer by sponging miR-124-3p. Cancer Cell Int 20: 40, 2020.

36. Jia P, Cai H, Liu X, Chen J, Ma J, Wang P, Liu Y, Zheng J and Xue Y: Long non-coding RNA H19 regulates glioma angiogenesis and the biological behavior of glioma-associated endothelial cells by inhibiting microRNA-29a. Cancer Lett 381: 359-369, 2016.

37. Hu Z, Cui Y, Zhou Y, Zhou K, Qiao X, Li C and Wang S: MicroRNA-29a plays a suppressive role in non-small cell lung cancer cells via targeting LASP1. Onco Targets Ther 9: 6999-7009, 2016.

38. Moore DD and Luu HH: Osteosarcoma. Cancer Treat Res 162: 65-92, 2014.

39. Sleijfer S, Rizzo E, Litière S, Mathijssen RHJ, Judson IR, Gelderblom H, Van Der Graaf WTA and Gronchi A: Predictors for doxorubicin-induced hematological toxicity and its association with outcome in advanced soft tissue sarcoma patients; a retrospective analysis of the EORTC-soft tissue and bone sarcoma group database. Acta Oncol 57: 1117-1126, 2018.

40. Wang D, Wang D, Wang N, Long Z and Ren X: Long non-coding RNA BANCR promotes endometrial cancer cell proliferation and invasion by regulating MMP2 and MMP1 via ERK/MAPK signaling pathway. Cell Physiol Biochem 40: 644-656, 2016.

41. He P, Zhang Z, Huang G, Wang H, Xu D, Liao W and Kang Y: miR-141 modulates osteoblastic cell proliferation by regulating the target gene of lncRNA H19 and lncRNA H19-derived miR-675. Am J Transl Res 8: 1780-1788, 2016.

42. Liu C, Pan C, Cai Y and Wang H: Interplay between long noncoding RNA ZEB1-AS1 and miR-200s regulates osteosarcoma cell proliferation and migration. J Cell Biochem 118: 2250-2260, 2017.

43. Orth MF, Cazes A, Butt E and Grunewald TG: An update on the LIM and SH3 domain protein 1 (LASP1): A versatile structural, signaling, and biomarker protein. Oncotarget 6: 26-42, 2015.

44. Wang LL, Wang L, Wang XY, Shang D, Yin SJ, Sun LL and Ji HB: MicroRNA-218 inhibits the proliferation, migration, and invasion and promotes apoptosis of gastric cancer cells by targeting LASP1. Tumour Biol 37: 15241-15252, 2016.

45. Gebeshuber CA, Zatloukal K and Martinez J: miR-29a suppresses tristetraprolin, which is a regulator of epithelial polarity and metastasis. EMBO Rep 10: 400-405, 2009.

46. Yu L, Liu S, Guo W, Zhang C, Zhang B, Yan H and Wu Z: hTERT promoter activity identifies osteosarcoma cells with increased EMT characteristics. Oncol Lett 7: 239-244, 2014.

47. Liu X, Fan Y, Xie J, Zhang L, Li L and Wang Z: Dehydroandrographolide inhibits osteosarcoma cell growth and metastasis by targeting SATB2-mediated EMT. Anticancer Agents Med Chem 19: 1728-1736, 2019.

48. Casola S, Pedone PV, Cavazzana AO, Basso G, Luksch R, d'Amore ES, Carli M, Bruni CB and Riccio A: Expression and parental imprinting of the $\mathrm{H} 19$ gene in human rhabdomyosarcoma. Oncogene 14: 1503-1510, 1997.

49. Wang L, Cai Y, Zhao X, Jia X, Zhang J, Liu J, Zhen H, Wang T, Tang X, Liu Y and Wang J: Down-regulated long non-coding RNA H19 inhibits carcinogenesis of renal cell carcinoma. Neoplasma 62: 412-418, 2015.

50. Chan LH, Wang W, Yeung W, Deng Y, Yuan P and Mak KK: Hedgehog signaling induces osteosarcoma development through Yapl and H19 overexpression. Oncogene 33: 4857-4866, 2014.

This work is licensed under a Creative Commons Attribution-NonCommercial-NoDerivatives 4.0 International (CC BY-NC-ND 4.0) License. 